\title{
Energy Efficient Relay Selection Algorithm for Virtual MIMO Cooperative Networks
}

\author{
Mohamad El Cheikh, Oluyomi Simpson and Yichuang Sun \\ School of Engineering and Computer Science \\ University of Hertfordshire, Hatfield, Hertfordshire, AL10 9AB, UK \\ E.C.Mohamad@herts.ac.uk,o.simpson@herts.ac.uk and y.sun@herts.ac.uk
}

\begin{abstract}
In this paper, we propose a distance based energy efficient multiple relay selection algorithm for cooperative virtual Multiple-Input-Multiple-Output (MIMO). The fundamentals of this method is to forward the source signal using the node which minimizes the end-to-end total path distance, such that the total energy cost per bit is reduced at the relay and at the source. An energy efficient multiple relay selection algorithm is proposed to minimize the energy cost per bit while achieving a target system performance in terms of $\mathrm{BER}$ at the destination. The core of the proposed relay selection method is selecting the node set that minimize the overall path lengths. We present the impact of the relay location and the constellation size for different MIMO configuration, and prove numerically that minimizing the sum of all path link length leads to lower energy consumption under the same performance requirement for MIMO, Multiple-Input-Single-Output (MISO) and Single-Input-Multiple-Output (SIMO) configuration. We compare the performance of MIMO, SIMO and MISO in terms of energy consumption and we present the results in terms of energy cost per bit against transmission distance. The results presented show that the proposed algorithm outperforms non optimized MIMO and traditional virtual MIMO communication in terms of energy consumption per bit for fixed rate and variable rate systems.
\end{abstract}

Index Terms-Cooperative Communications; Virtual MIMO; Relay Selection; Wireless Sensor Networks; Energy Efficiency.

\section{INTRODUCTION}

The demand for higher data rates communications over long transmission ranges was the primary motivation for the development of multiple-input-multiple-output (MIMO) systems used in modern wireless standards, as they support higher throughput under bad channel conditions [1]. Inspired by MIMO, virtual MIMO has been introduced to cooperative wireless network and has shown to be an effective technique to combat the fading nature of the wireless channel and improve system performance [2-4]. Virtual MIMO has several variations, the trivial case Single Input Single Output (SISO), Single-Input-Multiple-output (SIMO) and Multiple-InputSingle-Output (MISO). In cooperative-virtual MIMO, the transmitting wireless nodes and/or receiving wireless nodes do not necessarily have multiple antennas, but it is a group of single/multiple antenna transmitters and single/multiple antenna receivers, grouped together to form a cluster and act as a MIMO system at each side $[5,6]$ as shown in Figure 1. Although, virtual MIMO systems provide clear benefits at the application level, the design of virtual MIMO schemes introduce significant challenges such as synchronization and additional digital signal processing (DSP) [7-9]. Moreover, the enhancement in the network performance using virtual MIMO, add an energy penalty caused by additional circuit operations as more nodes are involved in the transmission [10]. Since the pioneering work in $[11,12]$, where the authors derived the energy consumption of virtual MIMO systems, the architecture proposed and the energy consumption affecting factors have made up the core of several works illustrating the performance of these schemes in cooperative wireless networks from different perspectives.

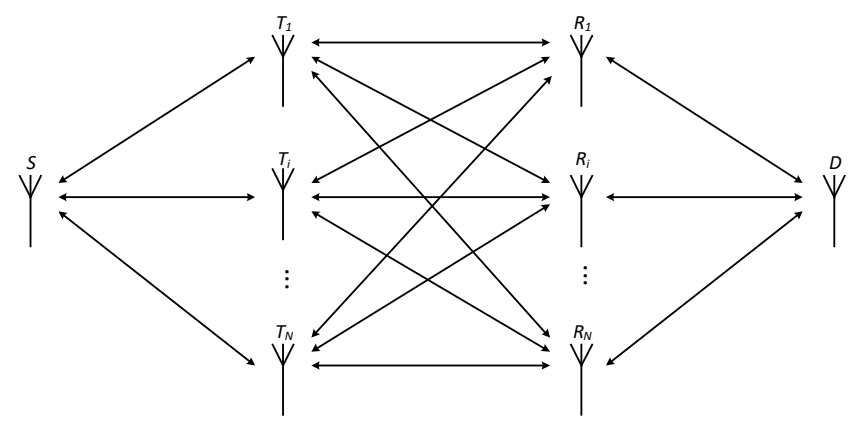

Figure 1. Virtual Cooperative MIMO System.

Virtual MIMO cooperative communication performance in terms of energy efficiency and consumption has been studied and investigated for fixed rate or variable rate system $[13,14]$. In [15], it has been proved that MIMO systems require less transmission energy than SISO systems for the same throughput requirements. This topic was deliberated in [16], showing that for a critical distance between transceivers, MIMO outperforms SISO in terms of energy efficiency. Consequently, it has been proved in [17] that nodes operating on MIMO structure and employing Space Time Block Codes (STBC) spend less energy than SISO systems. The most recent progress towards improving virtual MIMO in terms of energy efficiency is introducing relay selection schemes. Hence, under certain selection criteria the system performance in terms of energy efficiency can be improved, and several routing techniques and optimization schemes have been proposed in literature [18-22]. Decode and forward (DF) and amplify and forward (AF) energy efficiency performance was analyzed in [21], for various relay position subject to symbol error probability (SEP) at the destination. The authors presented the correlation between the energy cost and the transmission distance, and results prove that 
DF outperforms AF at long distance. In [22], cooperative MISO performance in terms of energy efficiency and delay has been investigated using a joint optimization strategy, and the proposed routing methods based on minimizing the end-to-end energy consumption showed better performance compare to SISO systems. In [23], the routing protocol selects the optimal transmission path that minimize the energy cost, and showed better performance when compared to SISO transmission in terms of the network lifetime. In [24], a finite feedback rate was used to determine the transmit power in clustered Wireless Sensor Networks (WSN) and the performance of the proposed algorithm extended the nodes serving time. In [25], energy efficiency in cooperative MISO was triggered using a selection function that determine the number of cooperative nodes in clustered WSN.

The proposed selection function, which combines the channel condition, residual energy and inter cluster distance, was shown to be more energy efficient than SISO or a nonselective approach. Moreover, the performance of cooperative MISO in multi-hop network was compared to other transmission techniques, and it was shown that single hop transmission outperforms multi-hop under certain distance threshold [26]. In addition of that, energy efficient cooperative MISO was approached using power allocation, where the authors proposed determining the transmit power using analytical calculation before the cooperative transmission [27]. In cooperative virtual MIMO, energy efficiency was triggered by proposing node selection for virtual MIMO formation such in [28].

To the best of our knowledge, distance based node selection has not been used as a relay selection metric to minimize the energy consumption in virtual MIMO networks. The contribution of this paper can be summarized as follows:

- We minimize the energy cost per bit by selecting the relay with minimum transmission path length.

- We investigate the constellation size and the impact on energy cost, thus we use the optimal modulation strategy jointly with the relay selection method.

- We offer adaptable solution benefiting from the RTS/CTS packet exchange, whereby the relay(s) adjust their transmit power to achieve the target BER.

- We offer an adaptive solution for systems with unknown relays number by switching between different MIMO configuration.

The rest of the paper is organized as follows: In Section II, the proposed scenario is given, which covers the system model, energy cost, impact of constellation size and the problem formulation. In Section III, the proposed relay selection algorithms for different MIMO configuration are presented. The performance of the proposed algorithm is illustrated through simulation using numerical values in Section IV. Finally, we conclude this paper in Section V.

\section{Proposed ScENARIO}

\section{A. System Model}

We consider the system shown in Figure 1, where two nodes communicate with the help of $\mathrm{N}$ relays, implementing either MIMO, SIMO or MISO configuration. In MIMO configuration, the communication consists of three stages, at the first stage the source transmits the message to $T$ transmitters nodes, which forwards the message to $R$ receivers nodes at stage two. At stage three, the receiver nodes forward the message to the destination. In MISO configuration, the source transmits at stage one, and $N$ relays forward the message at stage two. In SIMO configuration multiple sources transmit at stage one, and single relay forward the message to the destination at stage two. The communication implements the Time Division Multi Access (TDMA) scheme given in Figure 2, and the nodes get the channel information from the RTS/CTS packet exchange at the beginning of the transmission. We design $2 \times 2$ MIMO, $1 \times 2$ SIMO and $2 \times 1$ MISO schemes which can be expended to superior numbers, subject to system requirements.

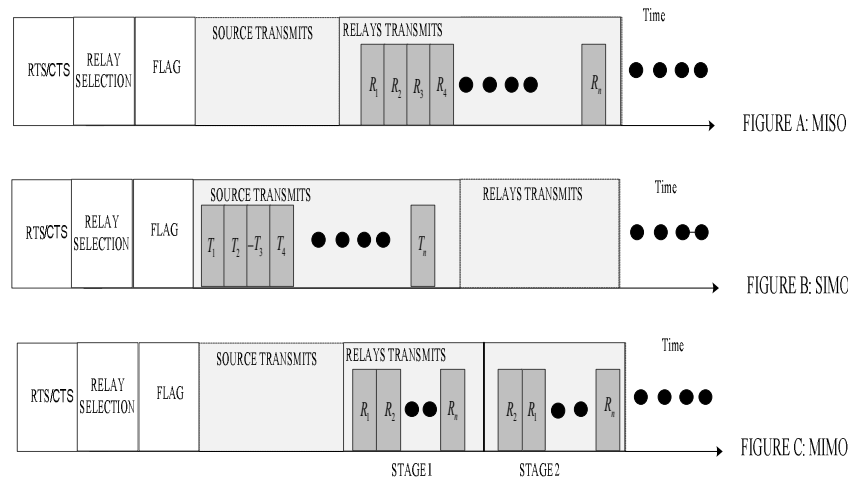

Figure 2. Cooperative Virtual MIMO TDMA Communication.

\section{B. Energy Cost}

We assume a Raleigh fading channel for all the links, where the transmit power falloff is proportional to the transmission distance following the path loss model given by

$P_{i j}=\bar{E}_{b} R_{b} G d_{i j}^{k}$

where $P_{i j}$ is the transmit power from node $i$ to node $j, \bar{E}_{b}, R_{b}$, $G, d_{i j}^{k}$ and $k$ are the average received energy per bit, bit rate, the power attenuation factor, the distance between transceivers, and the path loss. The average probability of bit error $P_{e}$ in a Rayleigh channel can be approximated as given in equation (2) for an SNR $y_{b}$ calculated as in equation (3).

$$
\begin{aligned}
\operatorname{BER}\left(E_{b}\right)= & \frac{4}{b}\left(1-\frac{1}{2^{b / 2}}\right)\left(1-\frac{1}{\sqrt{1+\sqrt{y_{b}}}}\right)^{N_{t} N_{r}}\left(\frac{1}{2^{N_{t} N_{r}}}\right) \\
& \left(\sum_{a=0}^{N_{r} N_{t}-1} \frac{\left(a+N_{r} N_{t}\right) !}{\left(a ! N_{r} N_{t} !\right)}\left(1+\frac{1}{\sqrt{1+\sqrt{y_{b}}}}\right)^{a}\right)
\end{aligned}
$$

$y_{b} \approx\left(\frac{E_{b}\left|h_{i j}\right|_{F}^{2}}{M_{t} M_{r} N_{0}}\right)^{-M_{t} M_{t}}$ 
where $M_{t}, M_{r}, \bar{E}_{b},\left|h_{i j}\right|_{F}^{2}$ and $N_{0}$ are the transmit antenna number, receive antenna number, energy received per bit, channel Frobenius matrix, and the noise spectral density, respectively. We evaluate the energy cost per bit for each MIMO configuration using the energy cost per bit for MISO. For MISO configuration, $E_{b}$ for $P_{e}$ is approximated as in [29] and is given by

$$
P_{e} \approx \varepsilon_{h}\left\{Q \sqrt{2 y_{b}}\right\} \approx\left(\frac{E_{b}}{M_{t} N_{0}}\right)^{-M_{t}}
$$

where $\varepsilon_{b}$ and $N_{0}$ are the channel expectation and the noise spectral density. As the source and destination are within the relay transmission range, for the same $P_{e}$ at both nodes, the required $E_{b}$ is the same. In a MIMO configuration each potential relay $E_{b}$ is calculated by

$$
E_{b}=\frac{M_{t} N_{0}}{P_{e}}
$$

The SNR is evaluated using equation (2) and $M_{r}$ is set to 1 . The sources use equation (5) to evaluate $E_{b}$ in SIMO and MISO configuration and at stage one and two of MIMO.

\section{Constellation Size Impact on Energy Consumption}

Although higher modulation minimizes the transmission time, an energy penalty is observed. Thus there a trade-off between the total energy consumption and the circuit energy consumption to achieve an optimal energy solution. Figure 3 illustrates the energy cost per bit in $\mathrm{dBm} \mathrm{mJ}$ against the transmission distance for different MIMO configuration for different modulation strategy. The optimal energy solution depends on the constellation size correlated to the transmission distance, whereby for MIMO configuration, the optimal constellation size is 32 , as it minimizes the energy consumption at a different transmission range. Moreover, MISO outperforms SIMO using the same constellation size at different transmission range. Furthermore, the impact of the constellations size in SIMO and MISO is negligible in correlation to the transmission distance compared to MIMO configuration for a long range. Thus, to achieve an energy efficient transmission switching between different MIMO configuration and applying adaptive modulation is the optimal transmission strategy. The optimal modulation strategy is given by

minimize $E_{i}(b)$

$$
\begin{array}{r}
\text { subject to } b-b_{\min }> \\
b_{\max }-b>0
\end{array}
$$

where $i$ is the node index. The node computes the energy budget $b$ for all different transmission deadlines.

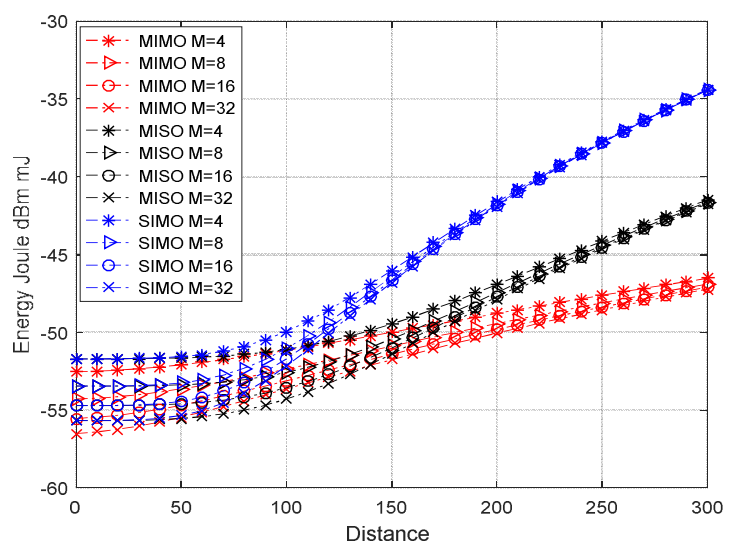

Figure 3. Energy Cost with Different Constellation Size

Using a convex optimization, the optimization problem is solved by the interior point method in the format given by [31]

minimize $E(b)$

subject to $E(b)>0$

The node start with minimum $b$, calculates the energy cost, and update $b$ in case the cost is minimized. Minimum and maximum value of $b$ is evaluated based on the transmission deadline requirements.

\section{Problem Formulation}

The probability of finding $k$ nodes out of $n$ to correctly decode the source signal follows the Bernoulli distribution and is given by

$$
P_{n}(k)=\left(\begin{array}{l}
n \\
k
\end{array}\right) P^{k}(1-k)^{n-k}
$$

where $P=r_{0} / R_{0}$ represent the probability of finding a successful relay within the source transmission range subject to a transmit desire power approximated based on the transmission range $r_{0}$ and the network radius $R_{0}$.

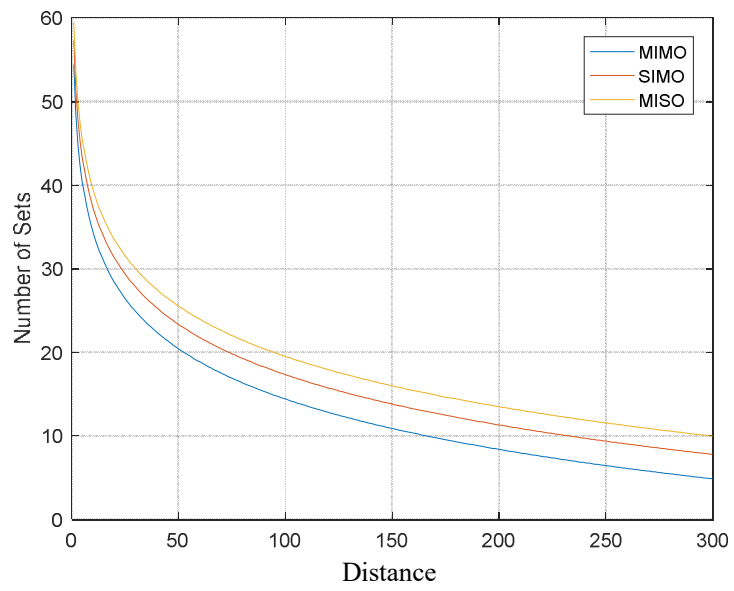

Figure 4. Number of Relays Set vs Distance 
Figure 4 considers the number of available relay sets, for different MIMO configuration against the transmission distance, and its clearly observed that the decrease in the set number is correlated with the transmission range. Thus, MIMO has the highest probability of successful transmission as the overall path is divided into shorter links. Under the same power constraint, the number of potential relay within the source transmission range increases which is due to the reduction of the required received power level at the receiver side.

\section{Proposed Relay Selection Algorithm}

\section{A. Optimization Policy}

The total transmission power cost $P_{t o t}$ allocated between the source and the relays is subject to a power constraint given by

$$
P_{s}+\sum_{1}^{n} P_{i} \leq P_{t o t}
$$

where $P_{s}$ is the source power and $P_{\text {tot }}$ is calculated using equation (5) based on direct transmission cost, and the sourcedestination path length. The distance based relay selection policy is given by

$$
\begin{gathered}
\operatorname{minimize} \quad E_{b} \\
\text { subject to } \sum_{1}^{i} d_{i}^{\alpha} \leq d_{t}^{\alpha} \\
d_{i} \geq d_{\min } \\
d_{i} \leq d_{\max }
\end{gathered}
$$

\section{B. MISO Relay Selection Algorithm}

For MISO configuration, the decoded relay set is the set of nodes that correctly decode the source messages given by

$$
D_{1}=\left\{P_{\text {Max_s }_{-} s} \bar{y}_{i}(S) \geq \bar{y}_{\text {th }}\left(b_{S R}\right)\right\}
$$

where $P_{M a x_{-} s}, \bar{y}_{i}(S)$ and $y_{t h}\left(b_{S R}\right)$ are the maximum transmit power at the source, the average received SNR at the node, and the SNR threshold, respectively, and $i$ is the node index. From the RTS message, nodes estimate $\bar{y}_{i}(S)$ and if equation (10) is satisfied, they listen to CTS message. At this stage, the second relay set that correctly decodes the CTS message is given by

$$
D_{2}=\left\{P_{\text {Max }_{-} D} \bar{y}_{i}(D) \geq \bar{y}_{t h}\left(b_{D R}\right)\right\}
$$

where $P_{M a x_{-} D}, \bar{y}_{i}(D)$ and $y_{t h}\left(b_{D R}\right)$ are the maximum transmit power at the destination, the received SNR at a node, and the SNR threshold for the target BER, respectively. Nodes set their timer using (12) and the node with minimum sum distance of both links has the smaller timer and the priority to send the Flag initiating itself as the successful relay. The sum distance is given by
$\Delta=\frac{d_{i}^{1}+d_{i}^{2}}{d_{l}} T$

where $d_{i}^{1}, d_{i}^{2}$ and $d_{l}$ are the relay-source and relay-destination path length, and $T$ is the transmission time slot allocated for each node to send the Flag initiating itself as the successful relay, and the algorithm is given in Table $\mathrm{I}$.

TABLE I

MISO Configuration Relay Selection Algorithm

\begin{tabular}{ll}
\hline \hline Algorithm 1: MISO Configuration Relay Selection Algorithm \\
\hline \hline Step 1: & $\begin{array}{l}\text { Candidates relay, switch to idle mode, at the } \\
\text { beginning of each transmission. }\end{array}$ \\
Step 2: & The source sends the RTS initiating data to transmit. \\
Step 3: & Candidate Relays compute (11). \\
Step 4: & The destination sends CTS. \\
Step 5: & Candidate Relays compute (12). \\
Step 6: & $\begin{array}{l}\text { Candidate Relays satisfying (11) and (12) compute } \\
\text { (13). }\end{array}$ \\
Step 7: & Relays use (5) and compute(6). \\
Step 8: & The source and the relay transmits following Figure \\
& 2.
\end{tabular}

\section{SIMO Relay Selection Algorithm}

For SIMO configuration, single relay forward multiple source signal, we define the decoded relay set as the set of nodes that correctly decode the sources messages given by

$$
D_{1}=\left\{P_{\text {Max_s }_{-}}^{j} \bar{y}_{i}\left(S_{j}\right) \geq \bar{y}_{t h}\left(b_{S R}\right)\right\}
$$

where $P_{\text {Max } \_s}^{j}, \bar{y}_{j}$ and $y_{t h}\left(b_{S R}\right)$ are the maximum transmit power at the sources, the average received SNR at the node, and the SNR threshold for a target BER, respectively. While $i$ and $j$ represent the receiving node index and the source index. The sources transmit RTS messages, and candidate relays execute equation (13). The relays decoding set listen to the destination CTS message and is minimized following the condition given in (14). The selection is competed in non-centralized manner, and the successful relay set their timer based on

$D_{2}=\left\{P_{M a x} \bar{y}_{i}(D) \geq \bar{y}_{t h}\left(b_{R D}\right)\right\}$

and the sum distance is given by

$\Delta=\frac{d_{i}^{1}+d_{i}^{2}+d_{i}^{3}}{d_{l}} T$

similar to MISO configuration case. The relay selection algorithm is given in Table II. 
TABLE II

SIMO Configuration Relay Selection Algorithm

\begin{tabular}{ll}
\hline \hline Algorithm 2: SIMO Configuration Relay Selection Algorithm \\
\hline \hline Step 1: & $\begin{array}{l}\text { Candidates relay, switch to idle mode, at the } \\
\text { beginning of each transmission. }\end{array}$ \\
Step 2: & The source sends the RTS initiating data to transmit. \\
Step 3: & Candidate Relays compute (14). \\
Step 4: & The destination sends CTS. \\
Step 5: & Candidate Relays compute (15). \\
Step 6: & $\begin{array}{l}\text { Candidate Relays satisfying (14) and (15) compute } \\
\text { (16). }\end{array}$ \\
Step 7: & $\begin{array}{l}\text { Relays use (5) and compute (6). } \\
\text { The source and the relay transmits following Figure } \\
\text { Step 8: }\end{array}$ \\
& 2.
\end{tabular}

\section{MIMO Relay Selection Algorithm}

For MIMO configuration, transmission consist of the following stages; the source transmits to the transmitting relay cluster, the transmitting relay cluster transmit to the receiving relay cluster, and finally the receiving relay cluster forwards the signal to the destination. Similar to SIMO and MISO communication mode, we identify two decoding set, defined as the source decoding set given by

$$
D_{1}=\left\{P_{M a x} \bar{y}_{i}(D) \geq \bar{y}_{t h}\left(b_{S R}\right)\right\}
$$

and the destination set given by

$$
D_{2}=\left\{P_{M A x} \bar{y}_{i}(D) \geq \bar{y}_{t h}\left(b_{R D}\right)\right\}
$$

The relay selection algorithm is given in Table III. The source selects the best set to form virtual MIMO at stage two, or request MISO configuration subject to relay availability by satisfying

$$
\begin{aligned}
& \operatorname{minimize} E_{b} \\
& \text { subject to } \sum_{1}^{i} d_{i}^{\alpha} \leq d_{t}^{\alpha}
\end{aligned}
$$

The major difference between the SIMO and MISO relay selection is the communication between relays at stage two. Therefore, potential relays have to estimate the virtual MIMO communication energy cost, to select the best grouping. At this stage the selection policy involve additional signaling overhead between candidate relays as the selection is done in an uncentralized manner.

To overcome additional signaling, we modify the selection policy such as each potential relay in the decoding sets transmit a 1-bit feedback after the RTS/CTS message exchange. Thus, all the potential relays and the destination have knowledge of the available sets. The source selects the best set to form virtual MIMO at stage two, or request MISO configuration subject to relay availability satisfying equation (19).
TABLE III

MIMO Configuration Relay Selection Algorithm

\begin{tabular}{ll}
\hline \hline Algorithm 3: MIMO Configuration Relay Selection Algorithm \\
\hline \hline Step 1: $\quad \begin{array}{l}\text { Candidates relay, switch to idle mode, at the } \\
\text { beginning of each transmission. }\end{array}$ \\
Step 2: & $\begin{array}{l}\text { The source sends the RTS initiating data to transmit. } \\
\text { Step 3: }\end{array}$ The destination sends CTS. \\
Step 4: & Candidate relays compute (17) and (18). \\
Step 5: & Relays Send one bit Feedback. \\
Step 6: & Source compute (19), and ACK. \\
Step 7: & MIMO or SIMO Formation. \\
Step 8: & Relays use (5) and compute(6). \\
Step 9: & The source and the relay transmits following Figure \\
& 2. \\
\hline
\end{tabular}

The source selects two relays from each set with a minimum two path lengths. Similarly, the destination follows the same procedure and predicts the communication strategy. This scenario is spotted in virtual MIMO, where the source transmits training sequence to the relays for channel estimation. The source transmits redundant bit to inform the successful relay of the communication strategy.

\section{Simulations Results}

To evaluate the performance of the proposed algorithm, we consider a fixed rate system with MQAM constellation and a symbol rate equal to the system bandwidth. We compare the energy consumption per bit of different MIMO configuration. using the parameters given in [30]. Thus, we consider a source node transmitting data to the destination using SISO, or SIMO configuration, or MISO or MIMO configuration. We compare the performance in terms of the energy consumption per bit. Figure 5 illustrates the performance of different MIMO configuration in terms of energy consumption per bit against the transmission distance in meters, and shows that different MIMO configurations outperforms SISO at different transmission distance. We replicate the same experiment for different MIMO configuration, using algorithm 1 - 3, which are presented in Table I - II, respectively and the results are presented in Figure 6. We assume 16-QAM fixed rate system without optimizing the constellation size.

It can be observed that MIMO outperforms MISO and SIMO with less than $1 \mathrm{~dB}$ at different transmission distances. This slightly comparable performance is due to additional energy cost of the overhead required as more relays cooperate in the transmission. However, the optimal energy solution is MISO as it provides the same performance in terms of total energy cost per bit with less complexity. We replicate the same experiment using the proposed algorithms for different MIMO configurations, and the results are shown in Figure 7. The Constellation size optimization improves the performance in terms of total energy consumption per bit for different configuration. 


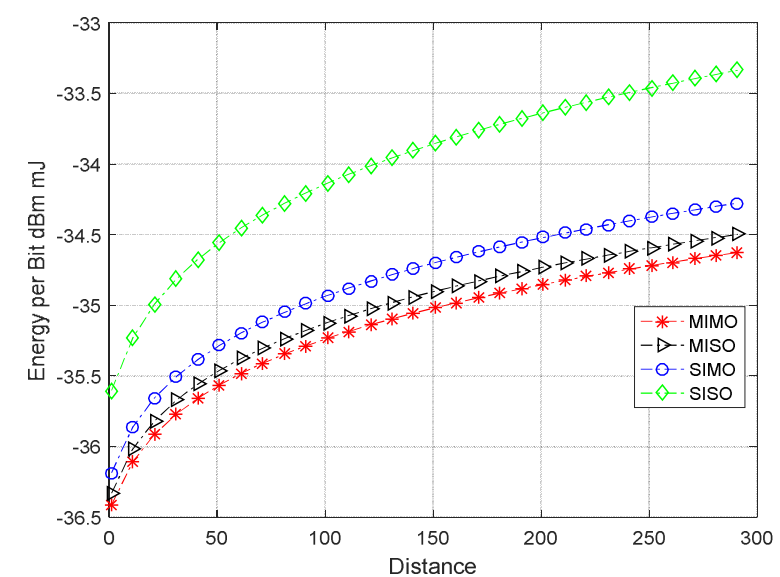

Figure 5. Energy Cost per Bit vs Distance (Relay Selection)

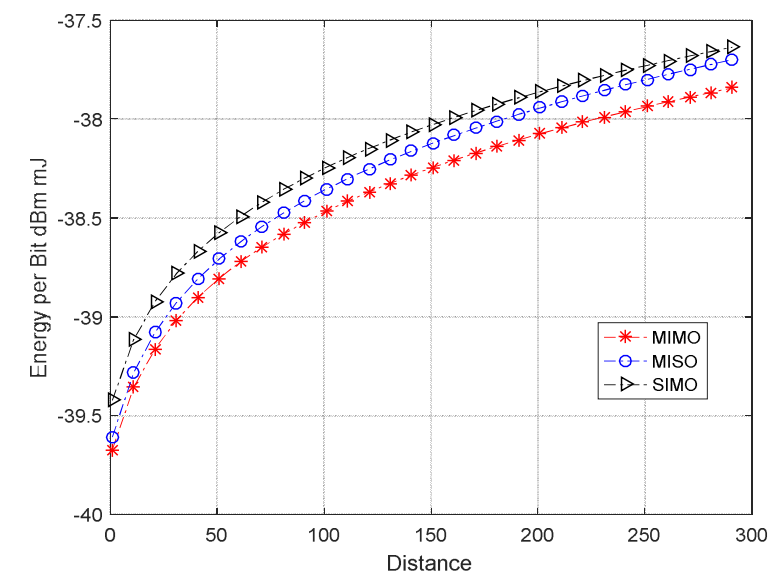

Figure 6. Energy Cost per Bit vs Distance (Relay Selection)

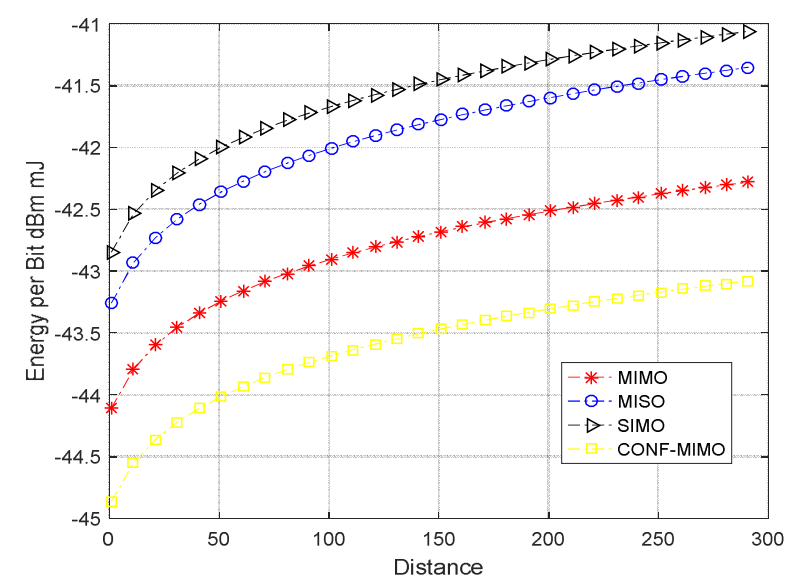

Figure 7. Energy Cost per Bit vs Distance, Optimized System

Moreover, a 2×2 MIMO (CON-MIMO) outperforms SIMO, and MISO by $2 \mathrm{~dB}$ at different transmission distance when switching between MISO and MIMO is applied. Thus, optimized MIMO is the optimal solution for the energy problem in a fixed rate system. In this configuration mode, further energy saving is achieved due to the decrease in relays by switching between MISO and MIMO subject to the system performance requirements. As a result, for short transmission range MISO achieve the same performance in terms of BER, consuming less energy when less circuit operations and signal receiving signal is required.

\section{CONCLUSION}

In this paper, an energy efficient relay selection algorithm for cooperative virtual MIMO was proposed. A distance based relay selection method is implemented taking into consideration the impact of the constellation size on energy consumption. We jointly optimize the constellation size with relay selection to achieve the optimal energy efficient transmission strategy. Furthermore, for MIMO configuration we employ switching between MIMO and SIMO based on the overall energy cost. We compared the performance of MIMO, SIMO and MISO in terms of energy consumption and we presented the results in terms of energy cost per bit against transmission distance. The proposed algorithm outperforms non optimized MIMO, and traditional virtual MIMO communication in terms of energy consumption in fixed rate system and variable rate system.

\section{REFERENCES}

[1] R. C. Chang et al., "Implementation of a High-Throughput Modified Merge Sort in MIMO Detection Systems," in IEEE Transactions on Circuits and Systems I: Regular Papers, vol. 61, no. 9, pp. 2730-2737, Sept. 2014.

[2] M. Rahmati and T. M. Duman, "Spectrally Efficient Alamouti Code Structure in Asynchronous Cooperative Systems," in IEEE Signal Processing Letters, vol. 21, no. 5, pp. 545-549, May 2014.

[3] F. A. Khan, et al., "Hybrid and Multi-Hop Advanced Zonal-Stable Election Protocol for Wireless Sensor Networks," in IEEE Access, vol. 7, pp. 25334-25346, 2019.

[4] X. Ma et al., "Adaptive Optimization of Multi-Hop Communication Protocol for Linear Wireless Monitoring Networks on High-Speed Railways," in IEEE Transactions on Intelligent Transportation Systems, vol. 20, no. 6, pp. 2313-2327, June 2019.

[5] S. Bameri, R. H. Gohary and S. Talebi, "Perfect Self-Interference Cancellation Based on Mode-Switching for Differential ChannelUnaware Two-Way Relay Networks," in IEEE Transactions on Wireless Communications, vol. 18, no. 11, pp. 5269-5283, Nov. 2019.

[6] M. Vajapeyam and U. Mitra, "Performance analysis of distributed spacetime coded protocols for wireless multi-hop communications, " in IEEE Transactions on Wireless Communications, vol. 9, pp. 122-133, 2010.

[7] S. Bameri, et al., "A Novel Self-Interference Cancellation Scheme for Channel-Unaware Differential Space-Time Two-Way Relay Networks," in IEEE Transactions on Wireless Communications, vol. 17, no. 2, pp. 1226-1241, Feb. 2018

[8] M. R. Avendi and H. Jafarkhani, "Differential Distributed Space-Time Coding With Imperfect Synchronization in Frequency-Selective Channels," in IEEE Transactions on Wireless Communications, vol. 14, no. 4, pp. 1811-1822, April 2015.

[9] G. Li, et al, "A Fuse-and-Forward Protocol for Two-Way Relaying Networks With Relay Having Its Own Broadcasting Information," in IEEE Communications Letters, vol. 19, no. 8, pp. 1450-1453, Aug. 2015.

[10] J. Kim, K. Kim and J. Lee, "Energy-Efficient Relay Selection of Cooperative HARQ Based on the Number of Transmissions Over Rayleigh Fading Channels," in IEEE Transactions on Vehicular Technology, vol. 66, no. 1, pp. 610-621, Jan. 2017.

[11] Z. Na, et al., "GFDM Based Wireless Powered Communication for Cooperative Relay System," in IEEE Access, vol. 7, pp. 50971-50979, 2019.

[12] S. Wang and T. Wu, "Stochastic Geometric Performance Analyses for the Cooperative NOMA With the Full-Duplex Energy Harvesting Relaying," in IEEE Transactions on Vehicular Technology, vol. 68, no. 5, pp. 48944905, May 2019.

[13] Z. Zhou, et al., "Wireless-Powered Cooperative Communications: PowerSplitting Relaying With Energy Accumulation," in IEEE Journal on 
Selected Areas in Communications, vol. 34, no. 4, pp. 969-982, April 2016.

[14] N. I. Zaman, K. M. A. A. Mamun, A. M. M. S. Uddin, and T. Kanakis, "Performance Analysis of MIMO Relaying Channels' Cooperative Diversity for Mobile Networks," in Second International Symposium on Data, Privacy, and E-Commerce, pp. 95-99, 2010.

[15] I. Dey, M. Majid Butt and N. Marchetti, "Throughput Analysis for Virtual MIMO WSNs Over Measured MIMO Channels," in IEEE Transactions on Instrumentation and Measurement, vol. 68, no. 1, pp. 297-299, Jan. 2019.

[16] V. K. Sachan, S. A. Imam, and M. T. Beg, "Energy-efficiency of Virtual cooperative MIMO techniques in wireless sensor networks," in International Conference on Computer Communication and Informatics, pp. 1-5, 2012.

[17] H. Chang and L. Wang, "A Low-Complexity Uplink Multiuser Scheduling for Virtual MIMO Systems," in IEEE Transactions on Vehicular Technology, vol. 65, no. 1, pp. 463-466, Jan. 2016.

[18] H. Xu, L. Huang, C. Qiao, W. Dai and Y. Sun, "Joint Virtual MIMO and Data Gathering for Wireless Sensor Networks," in IEEE Transactions on Parallel and Distributed Systems, vol. 26, no. 4, pp. 1034-1048, 1 April 2015.

[19] D. Jie, L. Danpu, W. Xin, and W. Huari, "An energy-efficient virtual MIMO transmission scheme for cluster-based wireless sensor networks," in 2010 IEEE 12th International Conference on Communication Technology, pp. 809-813,2010.

[20] Q. Gao, Y. Zuo, J. Zhang, and X. H. Peng, "Improving Energy Efficiency in a Wireless Sensor Network by Combining Cooperative MIMO With Data Aggregation," in IEEE Transactions on Vehicular Technology, vol. 59, pp. 3956-3965, 2010.

[21] N. Varshney and A. K. Jagannatham, "MIMO-STBC Based Multiple Relay Cooperative Communication Over Time-Selective Rayleigh Fading Links With Imperfect Channel Estimates," in IEEE Transactions on Vehicular Technology, vol. 66, no. 7, pp. 6009-6025, July 2017.
[22] L. Chen and G. Wei, "A Distributed Energy Efficiency Optimization Scheme for Cooperative Virtual MIMO System," in 2013 IEEE 78th Vehicular Technology Conference (VTC Fall), pp. 1-5,2013.

[23] K. G. Panda, D. Agrawal, and A. Hossain, "Virtual MIMO in wireless sensor network - a survey," in 2016 Online International Conference on Green Engineering and Technologies (IC-GET), pp. 1-4,2016.

[24] X. Jia, P. Deng, L. Yang and H. Zhu, "Spectrum and Energy Efficiencies for Multiuser Pairs Massive MIMO Systems With Full-Duplex Amplifyand-Forward Relay," in IEEE Access, vol. 3, pp. 1907-1918, 2015.

[25] G. Lim and L. J. Cimini, "Energy-efficient cooperative beamforming in clustered wireless networks," in IEEE Transactions on Wireless Communications, vol. 12, no. 3, pp. 1376-1385, March 2013.

[26] J. Zhang, L. Fei, Q. Gao and X. Peng, "Energy-Efficient Multihop Cooperative MISO Transmission with Optimal Hop Distance in Wireless Ad Hoc Networks," in IEEE Transactions on Wireless Communications, vol. 10, no. 10, pp. 3426-3435, October 2011.

[27] G. Zheng, T. Wei, and J. Li, "A MISO cooperative transmission algorithm for clustering wireless sensor network," in 2011 International Conference on Electronics, Communications and Control (ICECC), pp. 2433$2436,2011$.

[28] X. Chen, Z. Zhang and C. Yuen, "Adaptive Mode Selection in Multiuser MISO Cognitive Networks With Limited Cooperation and Feedback," in IEEE Transactions on Vehicular Technology, vol. 63, no. 4, pp. 16221632, May 2014

[29] Z. Huang, T. Yamazato, and M. Katayama, "Energy efficiency of cooperative MISO technique in multi-hop wireless sensor networks, " in 2008 International Conference on Intelligent Sensors, Sensor Networks and Information Processing, pp. 511-516, 2008.

[30] H. Wan, J. F. Diouris, and G. Andrieux, "Power allocation for virtual MISO cooperative communication in wireless sensor networks," in The 3rd European Wireless Technology Conference, pp. 69-72, 2010.

[31] S. Boyd and L. Vandenberghe, Convex Optimization. Cambridge, U.K.: Cambridge Univ. Press, 2003. 